\title{
Modelo de implementación del Sistema de Gestión de la Prevención de Riesgos Laborales en una industria láctea de Riobamba - Ecuador
}

\section{RESUMEN}

En Ecuador existen altos índices de accidentabilidad así como de enfermedades laborales, las empresas están obligadas por la normativa legal vigente a implementar sistemas de gestión en seguridad y salud ocupacional, pero no existe una metodología que permita su adecuada implantación y operación para mejorar la eficacia de la gestión preventiva en el sector lechero ecuatoriano.

El propósito del presente trabajo de investigación fue diseñar un modelo que permita implementar un Sistema de Gestión de la Prevención de Riesgos laborales en una industria Láctea de Riobamba en Ecuador. Para el diseño del modelo de implementación, se tomó como base las normas internacionales ISO 14001, decisión 584 del Instrumento Andino de Seguridad y Salud en el trabajo, resolución 957 y OHSAS 18001, Decreto ejecutivo 2393 , resolución N. ${ }^{\circ}$ C.D.513 de la normativa ecuatoriana legal aplicable, principalmente de los sectores Productivos, Trabajo y Salud, y la normativa internacional emitida por la Organización Internacional de Trabajo. Para la implementación del Sistema de Gestión de Prevención de Riesgos Laborales, se aplicó un esquema instruccional modular sustentado por actividades de capacitación en cada módulo. El despliegue de cada módulo está sujeto a la información durante el diagnóstico efectuado al inicio del proceso. La implementación exitosa del Sistema de Gestión impactó positivamente en los resultados organizacionales de la empresa PRASOL (Lácteos Santillán), en lo que corresponde al desempeño de seguridad y de salud ocupacional, mejorando su eficacia, los entornos laborales de los trabajadores y disminución la accidentabilidad laboral. Así mismo, sentó las bases para la obtención de la certificación en el Ministerio de Trabajo y en el Instituto Ecuatoriano de Seguridad Social.

Palabras clave: gestión, riesgos laborales, accidentabilidad, industria láctea

IMPLEMENTATION MODEL OF OCCUPATIONAL HAZARDS Prevention Management System on a dairy INDUSTRY IN Riobamba- ECUADOR

\section{ABSTRACT}

In Ecuador there are high rates of accidents and occupational diseases companies are required by current legislation to implement management systems in occupational safety and health, but there is not a methodology for its proper implementation and operation that will improve the effectiveness of preventive management in the Ecuadorian dairy sector.

The purpose of this research was to design a model to implement an Occupational Hazards Prevention Management System on a Dairy industry in Riobamba-Ecuador. To design the implementation model, it was taken as the basis of intemational standards ISO 14001, decision 584 of the Andean Instrument on Safety and Health at Work, resolution 957 and OHSAS 18001 Executive Order 2393, Resolution No. C.D. 513 of the Ecuadorian legal regulations applicable, mainly in productive sectors, labor and health, as well as international standards issued by the International Labour Organization. To implement the Occupational Health and Safety Management System, a modular scheme supported by instructional training activities in each module was applied. The deployment of each module is subject to information during the diagnosis made at the beginning of the implementation process. The successful implementation of the management system caused a positive impact on organizational performance of the company PRASOL (Dairy Santillan), as it pertains to the performance of safety and occupational health, improving their efficiency, work environments for workers and decrease the labor accident. So also it laid the foundation for obtaining certification in the Ministry of Labour and the Ecuadorian Social Security Institute. Keywords: prevention management, occupational hazards, work accidents, dairy industry

\section{INTRODUCCIÓN}

TEOnILA García ZaPata***

Conforme el avance de la historia y las actividades de los individuos, los riesgos laborales han existido siempre y se han presentado a la par con el desarrollo de la ciencia y tecnología, los riesgos han tomado importancia, determinando enfermedades ocupacionales y accidentes con lesiones significativas. Vidal, Santiago y otros (2015) afirman que la prevención de riesgos laborales trata de proteger el derecho de las personas a su integridad física y su salud, para ello la sociedad trata de dictar normas que modulen el comportamiento de los actores, empresarios y trabajadores en el mundo del trabajo, y mantener bajo control los riesgos que el trabajo conlleva y a partir de aquí lograr el objetivo de proteger a las personas. La Organización Internacional del Trabajo (OIT), estima que los costos de los accidentes y enfermedades ocupacionales alcanzan hasta el $10 \%$ del Producto Interno Bruto (PIB). Según estos datos, cada día mueren 6300 personas a causa de accidentes o enfermedades relacionadas con el trabajo, más de 2.3 millones de muertes por año. Anualmente ocurren más de 317 millones de accidentes en el trabajo, muchos de estos accidentes resultan en ausentismo laboral. Por lo que existe conciencia mundial sobre los riesgos laborales, su magnitud y las consecuencias de los accidentes, las lesiones y las enfermedades relacionadas con el trabajo. Pozo (2009 citado en Cedeño, 2011), sostiene que según el Instituto Ecuatoriano de Seguridad Social (IESS) en Ecuador 10,5 trabajadores mueren cada día, existen 2751 muertes por año, con 158760 accidentes de trabajo y 18900 enfermedades profesionales.

Actualmente el Ecuador se acoge a la resolución 957 del reglamento del Instrumento Andino de Seguridad y Salud en el Trabajo (2005) a través del IESS, que establece las políticas de prevención de riesgos laborales, seguridad y salud en centros de trabajo. La publicación realizada por el Ministerio de Relaciones Laborales (MRL, 2014), acuerdo entre el Ministerio de Relaciones Laborales y el Instituto Ecuatoriano de Seguridad Social para expedir el instructivo de implementación del Sistema Nacional

* Magíster en Gestión de la Producción, Doctor en Ingeniería Industrial, Docente Investigador Titular de la Facultad de Ingeniería de la Universidad Nacional de Chimborazo, Riobamba, Ecuador. E-mail: pvillacres@unach.edu.ec

** Magíster en Diseño Gestión y Evaluación de Proyectos Sociales y Productivos, Docente Investigador Titular de la Facultad de Ingeniería en Industrias Pecuarias de la Escuela Superior Politécnica de Chimborazo, Riobamba, Ecuador. E-mail: dbano@espoch.edu.ec

*** Doctora en Ingeniería Industrial, Profesor Principal de la Facultad de Ingeniería Industrial de la UNMSM. E-mail: teogaza57@yahoo.es 
de Gestión de Prevención de Riesgos Laborales (SGP), cumplen con el mandato constitucional de proveer ambientes de trabajos seguros y saludables, acordes al plan nacional del buen vivir; generando prácticas saludables, previniendo y minimizando los riesgos del trabajo, incrementando niveles de producción en las empresas. Esto ha conllevado que las mencionadas entidades del estado estén desarrollando actividades verificadoras del cumplimiento de la normativa nacional basado en la resolución C.D.333, decreto ejecutivo 2393,resolución C.D. 390 y C.D. 513 que establece el Sistema de Auditoria de Riesgos del Trabajo (SART) para las industrias ecuatorianas, como medio de verificación del cumplimiento de la normativa legal, sobre las responsabilidades de la Dirección del Seguro General de Riesgos del Trabajo dirigida a la aplicación futura de los programas de auditorías de riesgos del trabajo a las organizaciones.

La gestión adquiere un papel fundamental como herramienta para conseguir la reducción y eliminación de los riesgos. Según las normativas de seguridad (Enríquez y Sánchez, 2008). la gestión se define como: "actividades coordinadas para dirigir y controlar una actividad u organización"; entonces siguiendo este enfoque y relacionándolo a los riesgos laborales, la misma norma define la gestión del riesgo como: "aplicación sistemática de políticas, procedimientos y prácticas de gestión para analizar, valorar y evaluar los riesgos". El modelo de gestión de prevención sugerido por el estado ecuatoriano se fundamenta en el "Reglamento del Instrumento Andino de Seguridad y Salud en el Trabajo" (2005), capítulo I gestión de la seguridad y salud en el trabajo (artículo 9, decisión 584). Mismo que considera las siguientes dimensiones: La gestión administrativa, gestión técnica, gestión del talento humano y procedimiento y programas operativos básicos. Estos son los fundamentos sobre los cuales el Dr. Luis Vásquez Zamora, desarrolló el Modelo Ecuador para la gestión de la seguridad y salud ocupacional, pero su implementación requiere de un modelo instruccional, que facilite la implantación de todos sus componentes en forma técnica, eficaz y con mejora continua, haciéndose necesario entonces determinar las correlaciones de éstas con el modelo propuesto.

En el Ministerio de Agricultura, Ganadería y Pesca del Ecuador (MAGAP), existen 28 plantas procesadoras de leche registradas en las zonas de mayor producción entre ellas las de la provincia de Chimborazo-Riobamba. La capacidad total instalada de estas es de 1380.800 litros (Vargas, 2010). Este creciente desarrollo de la industria lechera requiere de maquinaria y equipo, fundamentalmente en acero inoxidable, para procesos de Pasteurización en HTST (High Temperature Short Time) o de larga vida en UHT (Ultra High Temperature), calderos para la generación de vapor en todos sus procesos, separadores centrífugos, homogenizadores, intercambiadores de calor, sistemas de refrigeración y conservación, personal calificado, generándose condiciones peligrosas de riesgo en la fuente, en el medio y en el receptor, ya que las empresas lecheras en Riobamba no han implementado programas de prevención de riesgos y peor aún, evaluación de factores de riesgos sean estos físicos, mecánicos, ergonómicos, psicosociales, químicos, biológicos o de riesgos mayores debido al desconocimiento sobre la implementación y diseño del sistema de gestión de prevención de riesgos, incrementando así, los riesgo de accidentes y enfermedades ocupacionales . Peula, (2014), afirma que la gestión de la prevención está directamente relacionada con la seguridad y salud laboral en plantas de envasado de productos lácteos, pues al evitar accidentes laborales se evitan posibles contaminaciones alimentarias con una productividad mayor, por la reducción de paros imprevistos. Un correcto funcionamiento de la seguridad y salud laboral repercute en una empresa láctea más eficiente y competitiva. Según Azcuénaga (2004), en el siglo XXI la globalización de mercados y de nuevos requerimientos de competitividad industrial, las empresas necesitan una metodología que facilite la implantación, de calidad y seguridad planteada a medio y largo plazo. La hipótesis del trabajo de investigación fue que el Sistema de Gestión de la Prevención de Riesgos laborales implementado con el modelo propuesto mejora la eficacia de la Gestión en Seguridad Ocupacional, así como disminuyen el índice de frecuencia de accidentes en una industria láctea de Riobamba- Ecuador.

\section{MATERIALES Y MÉTODOS}

Se seleccionó a la empresa de productos lácteos Santillán, para aplicar el modelo de implementación del sistema de gestión de la prevención de riesgos laborales (MSGPRL) propuesto, por ser la más representativa, en la Provincia de Chimborazo, se aplicó el Sistema de Auditorías de Riesgos del Trabajo para establecer el diagnóstico inicial de la prevención de riesgos laborales en el año 2014, luego de 12 meses de implementación del sistema de gestión en seguridad y salud laboral, se realizó la auditoria final con la matriz SART, resolución C.D. 333 del Estado Ecuatoriano, que permitió evaluar los índices de eficacia y accidentabilidad. 
Se diseñó el constructo para aplicar al personal de la empresa en mención, estableciéndose el modelo multifactorial correlacional entre el modelo de implementación propuesto y los componentes del sistema de gestión en seguridad y salud del modelo Ecuador.

\section{APLICACIÓN DE LA METODOLOGÍA}

En esta investigación se desarrolló en primera instancia el Modelo de Implementación del Sistema de Gestión de la Prevención de Riesgos Laborales, considerando la normatividad en materia del sistema de gestión que se desea integrar, las expectativas de las partes interesadas en correspondencia con los intereses de clientes y entidades reguladoras estatales. Resultan relevantes en el procedimiento los grupos de interés en el ámbito de la gestión de la seguridad, conformado por: la alta dirección, para establecer estrategias, aportar recursos necesarios, ayudar a la toma de decisiones y la solución de los problemas; los especialistas, que atienden los sistemas en análisis, se encargan de asesorar a la alta dirección, su formación y competencias son esenciales; $y$, los empleados, mandos intermedios, que son los que crean valor en los procesos y se encargan de aplicar los procedimientos que sustentan cualquier sistema de gestión. Esta concepción toma como base el sistema de gestión de la seguridad ocupacional el cual constituye un sistema integrador por excelencia (Díaz, 2013) al estar vinculado directamente con la elaboración de productos o generación de servicios bajo entornos laborales seguros. De ahí que, el enfoque principal de gestión seleccionado sea el del ciclo de mejora de Deming, que se aprecia en la Figura 1, "Planificar-Hacer-Verificar-Actuar" (PHVA) el cual tiene carácter dinámico y puede desarrollarse dentro de cada proceso de la organización y en el sistema prevención de riesgos, como un todo; está íntimamente asociado con la planificación, implementación, control y mejora continua, en todos los procesos. Estas fases pueden desarrollarse de forma simultánea y encontrarse en diferentes etapas del ciclo de gestión; con enfoque de procesos integrados y su accionar como una espiral en desarrollo, dado el carácter cíclico del proceso en mención. Como transformación esencial se logra la gestión de riesgos de seguridad y salud ocupacional integrados a la gestión de la empresa. Desde el punto de vista metodológico, se presentan como componentes del sistema: el diagnóstico, que permite caracterizar la situación de la gestión de la seguridad laboral en función de los requisitos establecidos por las normas correspondientes y los resultados de su gestión.

. La aplicación del modelo de implementación que se propone, requiere como premisas la normativa legal vigente, especificadas en el acápite anterior, cuyas componentes abarcan las gestiones administrativa, técnica, del talento humano y de procesos operativos básicos. De igual forma se precisa el apoyo financiero que permita su aplicación. Según Peltier (2013), el ciclo de mejora continua, permite establecer modelos de gestión de procesos como se aprecia en la Figura 2, cuyos principios interactúan en la gestión e implementación de los sistemas de seguridad ocupacional.

\section{Descripción del modelo propuesto: Fases y pasos que lo componen}

En la Figura 2, se representa el procedimiento propuesto, dividido en cuatro fases, cada una de ellas persigue un objetivo y se aplican distintas herramientas de acción para lograr resultados parciales, que serán argumentos que fundamenten a la siguiente fase. En la Fase I, se identifican los procesos que luego conformarán el mapa de procesos; se define la línea base o punto de partida del estudio, el cual arrojará como resultado estrategias de mejoramiento. En la Fase II, se explicarán la panificación y programación para implementación del sistema de gestión en función del diagnóstico inicial, que permitirá obtener la propuesta de integración, con sus registros y diagramación en la Fase III se plantea la forma como se realizará la implantación, según los componentes del modelo de gestión de prevención de riesgos laborales; bajo el proceso Planificar $(P)$; Hacer (H); Verificar (V); Actuar (A) y, en la Fase IV se diseñan los indicadores de gestión, se verificará el cumplimiento de los requisitos del sistema de gestión que aportará a mejorar la responsabilidad social corporativa.

\subsection{Actividades Complementarias}

El desarrollo del modelo multifactorial correlacional, implicó el diseño del constructo, la prueba piloto estableció su confiabilidad, mediante el Alfa de Cronbach, indicándose en qué medida las diferencias individuales de los puntajes en un test pueden ser atribuidos a las diferencias "verdaderas" de las características consideradas. (Rivas y Giraldo, 2015). El coeficiente alfa promedio de Cronbach fue $88,6 \%$ de fiabilidad. La validez se realizó con análisis factorial, en dónde la medida de adecuación muestral de Kaiser-Meyer-Olkin resulto alto $(K M O=0,890)$ esto señala que el análisis factorial se considera pertinente, la determinante 
Figura 1. Propuesta del Modelo de Implementación de la Gestión de la Prevención de Riesgos Laborales MSGPRL.

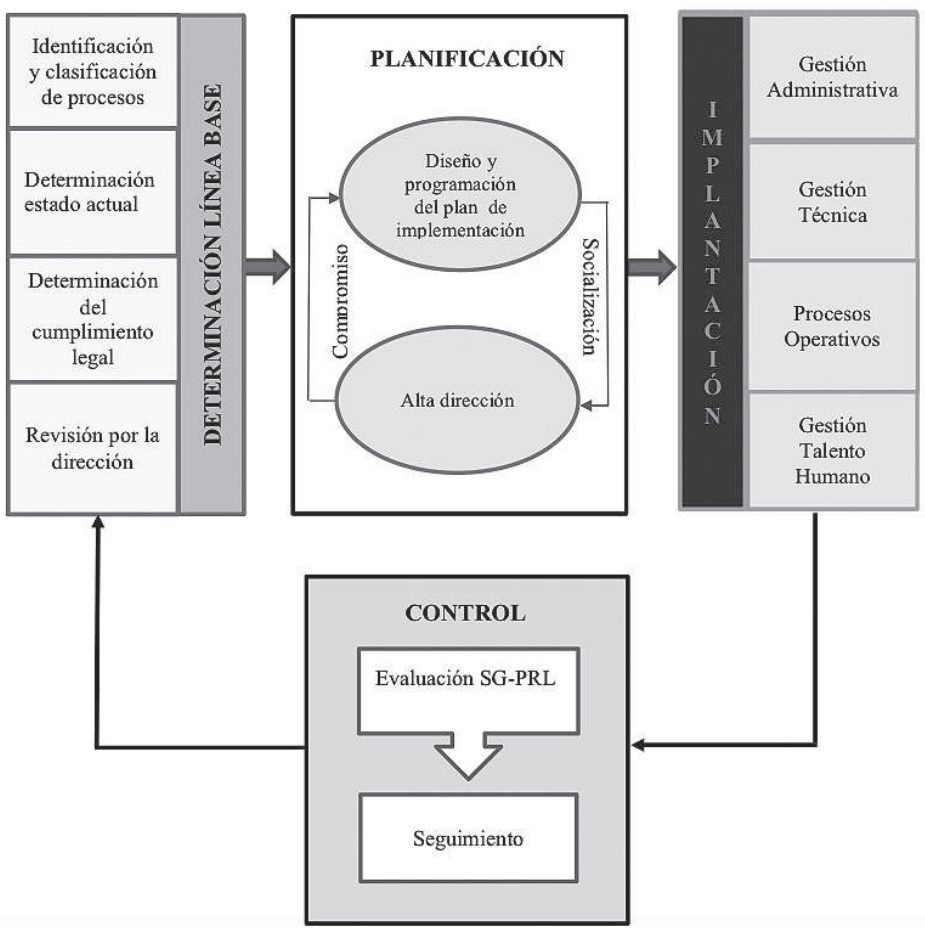

Fuente: Elaboración propia.

Figura 2. Modelo de Implementación de la gestión de la prevención de riesgos laborales.

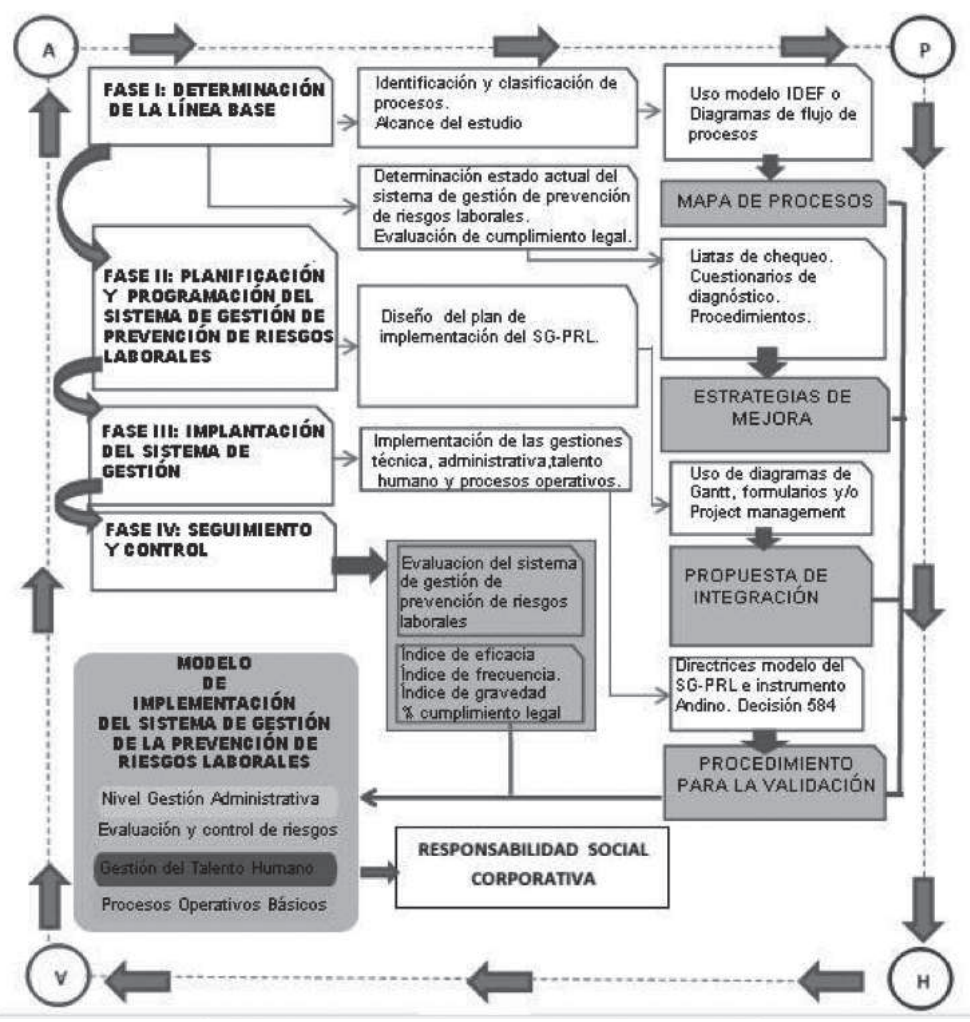

Fuente: Elaboración propia. 
de la matriz de correlaciones es próxima a cero (0.000), lo cual es bueno desde el punto de vista de la idoneidad del análisis. El método de rotación Varimax minimizó el número de factores a las cuatro gestiones del modelo Ecuador. Luego de lo cual se aplicó el instrumento a todos los empleados y trabajadores de la empresa en estudio.

\section{ANÁLISIS ESTADÍSTICO}

El análisis estadístico fue realizado aplicando estadística descriptiva, se usó el instrumento de "check list" del sistema de auditoria de riesgos del trabajo. El programa estadístico Informático SPSS Statistics 23.0, con sus pruebas no paramétricas, como la prueba de Wilcoxon, usados para verificar la diferencia significativa de antes y después de la implementación del MSGPRL en la empresa investigada con un nivel de significancia $\alpha=0,05$

\subsection{Resultados}

\section{Fase I. Determinación de la línea base:}

En la Figura 3, se aprecia la situación inicial de la gestión preventiva en la empresa investigada.

Se debe evaluar el índice de eficiencia del Sistema de Gestión de la Seguridad y Salud en la empresa, para lo cual se establece la siguiente expresión según Resolución C.D.390 del reglamento del seguro general de riesgos del trabajo.

$$
\begin{aligned}
& \mathrm{IEF}=\frac{\left(\mathrm{N}^{\circ} \text { elementos auditados integrados/implantados }\right)}{\left(\mathrm{N}^{\circ} \text { Total de elementos aplicables }\right)} * 100 \\
& \mathrm{IEF}=\frac{13.323}{25}=53.29 \%
\end{aligned}
$$

De acuerdo al diagnóstico realizado podemos determinar que la gestión técnica requiere atención inmediata con un $35 \%$ de eficacia, la empresa cumple en un índice de eficacia total de 53,29\% valor que de acuerdo a la Resolución C.D.333 del IESS, Art 11 inciso 1.2, es considerado como insatisfactorio y se debe implementar el modelo del Sistema de Gestión de Seguridad y Salud del Trabajo que mejore este indicador. El índice de frecuencia de accidentes es 19 en el año 2014, es decir elevado, respecto del valor referencial de 5 en industrias de bebidas y alimentos en el Ecuador, según datos del Instituto Ecuatoriano de Seguridad Social. Lo que indica un bajo nivel de seguridad en la organización.

\section{Fase II. Planificación y programación}

Se diseñó el plan de implementación, del SG-PRL, durante el año 2014, para su correspondiente

Figura 3. Diagnóstico del SART en la fábrica de lácteos Santillán - PRASOL.

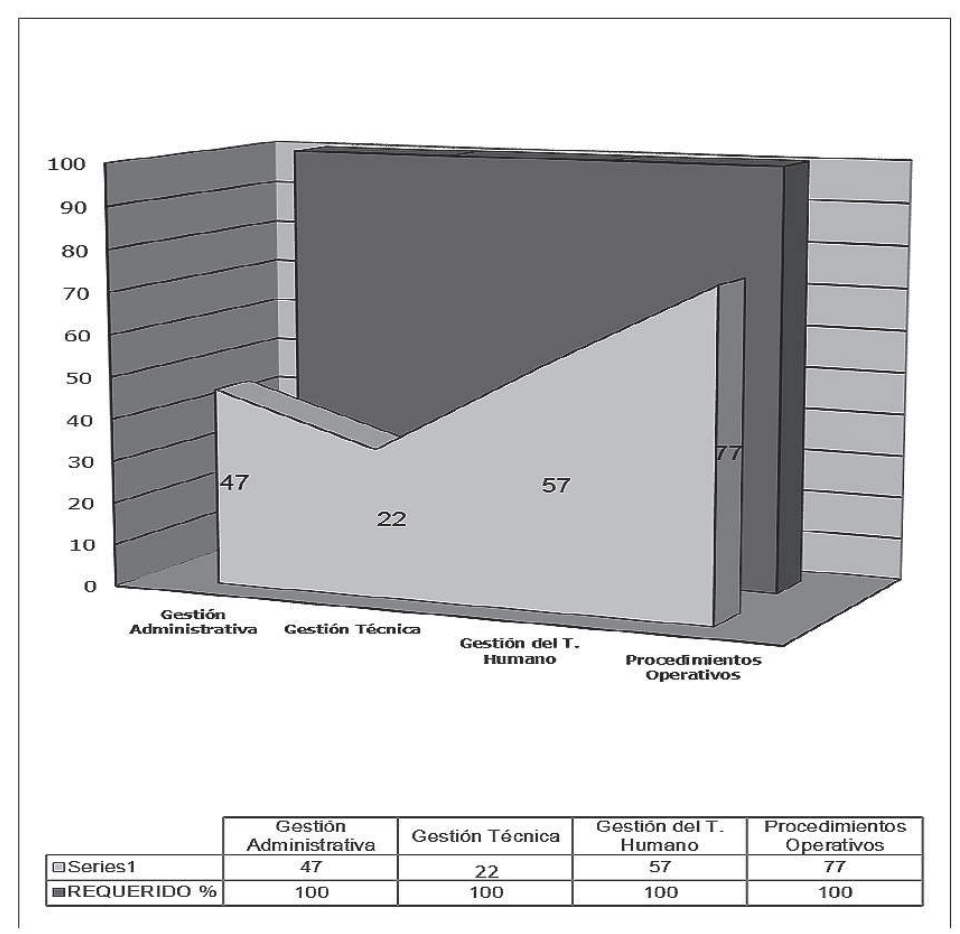

Fuente: Elaboración propia. 
evaluación en 2015, cuyos resultados se aprecian en las siguientes etapas del modelo propuesto.

\section{Fase III. Implantación}

En la Figura 4, se evidencian los resultados de la gestión administrativa, técnica, de talento humano y procesos operativos con la metodología propuesta, cuyos resultados incrementaron el porcentaje de eficacia y cumplimiento en seguridad laboral en toda la organización, $83 \%$, 95\%, 98\%,100\%, respectivamente.

\section{Fase IV. Seguimiento y control}

Una vez implementado el SG-PRL, se procede a aplicar la matriz SART, para evaluarlo considerando los indicadores de eficacia (IEF), frecuencia de accidentes (IF), índice de gravedad (IG), para finalmente establecer cómo se relaciona el modelo propuesto, con las dimensiones de seguridad y salud ocupacional del modelo Ecuador, cuyos datos se expresan en la Tabla 1.

El índice de eficacia (IE) del sistema de gestión implementado es satisfactorio:

$\mathrm{IEF}=\frac{23.38}{25}=93.52 \%$

La estadística de accidentabilidad en la fábrica de Lácteos Santillán - PRASOL en 2015, presentada en la Tabla 1 y Figura 6, después de implementar el SG-PRL, debe ser remitida anualmente al Seguro General de Riesgos del Trabajo

Con el modelo de implementación desarrollado, el MSGPRL incrementó el índice de eficacia al 93,59 \%, disminuyó el índice de frecuencia de accidentes anual a 3,3 y el de gravedad a 6,7en el año 2015 , con un decrecimiento mensual como lo establece la ruta táctica establecida y los valores reales alcanzados con su implantación.

\subsection{Comprobación estadística}

Para Rubio y Berlanga (2012), muestras dependientes o relacionadas, se refieren a las provenientes de un universo muestral, a las que se aplicará un plan experimental, mediante el cual se espera un cambio, de manera que en el análisis de las observaciones existen dos períodos: antes y después del tratamiento este es el típico caso de contraste de medias para grupos relacionados. Se aplicó la prueba de Kolmogorov-Smirnov para una muestra como procedimiento de "bondad de ajuste", que permite medir el grado de concordancia existente entre la distribución de un conjunto de datos y una distribución teórica específica. Al comprobar el nivel de significación, los índices de frecuencia de accidentes son menores que 0.05, por tanto la distribución no es normal (nivel de significación 0.004 y 0.000 respectivamente).

Para establecer si en efecto existe una mejora del índice de frecuencia de accidentes, se utiliza una prueba no paramétrica que permita efectuar un test de los rangos con signo de Wilcoxon comparando dos muestras de frecuencia de accidentes relacionadas y determinar si existen diferencias entre ellas. Además de considerar el sentido de las diferencias de las puntuaciones contempla también la magnitud de las mismas y así determinar si efectivamente el sistema de gestión de la prevención de riesgos laborales implementado con el modelo propuesto disminuye la accidentabilidad en una industria láctea de Riobamba- Ecuador.

Se comprueba que en los resultados del caso investigado, sí hay diferencias significativamente

Figura 4. Evaluación del sistema de gestión implementado.

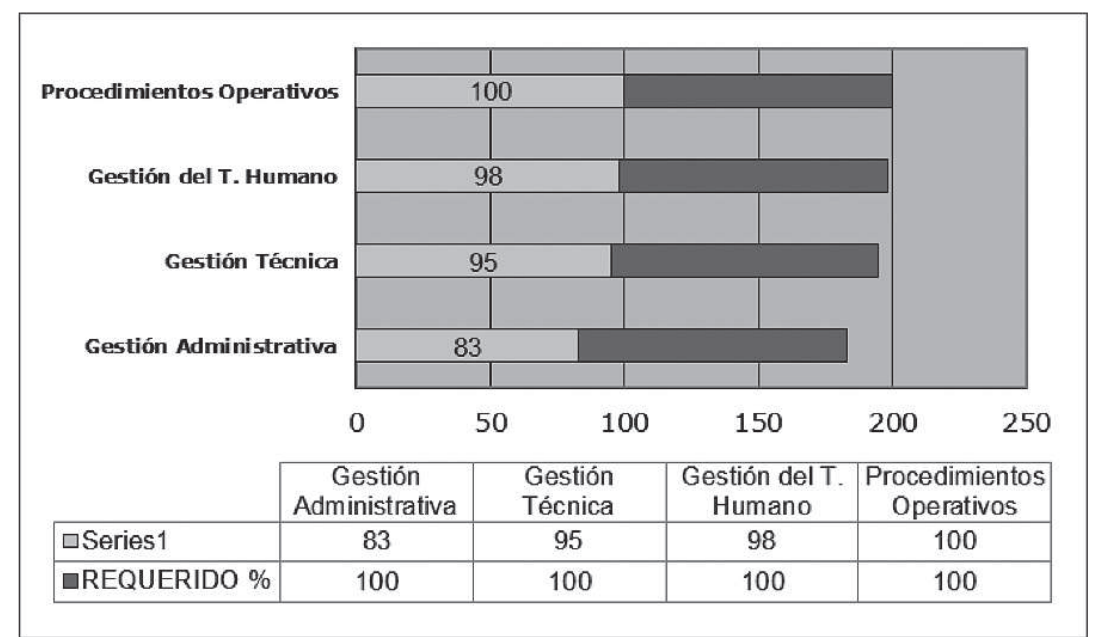

Fuente: Elaboración propia. 
Tabla 1. Datos Estadísticos de Accidentabilidad 2015

\begin{tabular}{|c|c|c|c|c|c|c|c|}
\hline & & & & & & & \\
\hline & T & & AD & 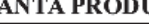 & & & \\
\hline MES & $\begin{array}{c}\text { No. } \\
\text { TRABAJADORES }\end{array}$ & $\begin{array}{c}\text { No. } \\
\text { ACCIDENTES }\end{array}$ & $\begin{array}{c}\text { No. DÍAS } \\
\text { PERDIDOS POR } \\
\text { LOS }\end{array}$ & $\begin{array}{c}\text { No. DÍAS } \\
\text { TRABAJADOS }\end{array}$ & $I F a=\frac{\mathbb{N}^{0} A C C^{*} 200000}{H H T}$ & $I G a=\frac{T^{2} 200000}{H H T}$ & $\mathbb{R}=\frac{\mathbb{1 0}}{\mathbb{F}}$ \\
\hline & DE PLANTA & & ACCIDENTES & AL MES & $\begin{array}{c}\text { INDICEDE } \\
\text { FRECUENCIA }\end{array}$ & $\begin{array}{l}\text { TINDICEDE } \\
\text { GRAVEDAD }\end{array}$ & $\begin{array}{l}\text { TASADE } \\
\text { RIESGO }\end{array}$ \\
\hline \begin{tabular}{|l|} 
Enero \\
\end{tabular} & 49 & 0 & 0 & 25 & 0,0 & 0,0 & 0 \\
\hline Febrero & 49 & 0 & 0 & 26 & 0,0 & 0,0 & 0 \\
\hline Marzo & 49 & 0 & 0 & 26 & 0,0 & 0,0 & 0 \\
\hline Abril & 49 & 0 & 0 & 26 & 0,0 & 0,0 & 0 \\
\hline Mayo & 49 & 0 & 0 & 26 & 0,0 & 0,0 & 0 \\
\hline Junio & 45 & 0 & 0 & 26 & 0,0 & 0,0 & 0 \\
\hline \begin{tabular}{|l} 
Julio \\
\end{tabular} & 45 & 0 & 0 & 26 & 0,0 & 0,00 & 0 \\
\hline Agosto & 45 & 0 & 0 & 26 & 0,0 & 0,00 & 0 \\
\hline Septiembre & 49 & 0 & 0 & 26 & 0,0 & 0,0 & 0 \\
\hline Octubre & 49 & 0 & 0 & 26 & 0,0 & 0,0 & 0 \\
\hline \begin{tabular}{|l|} 
Noviembre \\
\end{tabular} & 49 & 1 & 2 & 26 & 19,6 & 39,2 & 2 \\
\hline Diciembre & 49 & 1 & 2 & 25 & 20,4 & 40,8 & 2 \\
\hline TOTAL & 48 & 2 & 4 & 310 & 3,3 & 6,7 & \\
\hline SIGNIFICA & IDO DE ÍNDICI & SS ESTADÍST & ICOS & & & & \\
\hline IF = Indice de & frecuencia = Indic & a la cantidad de & e accidentes inc & citantes, mas & una jomada de $t$ & rabajo perdida. & \\
\hline$=$ Índice $c$ & lad: E: & dida uti & para indicar & d de las & curridas & ent & \\
\hline
\end{tabular}

Fuente: Elaboración propia.

Figura 5. Comparaciones índices de frecuencia de accidentes anual.

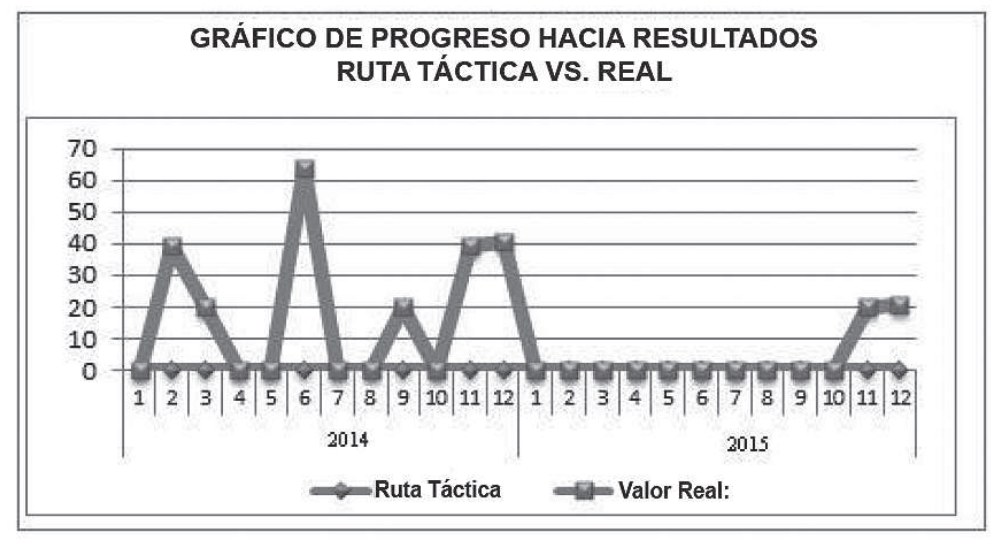

Fuente: Elaboración propia.

efectivas, ello implica el poder afirmar que la implementación del Sistema de Gestión de Prevención de Riesgos laborales, en la industria láctea analizada disminuye el índice de frecuencia de accidentes laborales, tal como registra la Tabla 2. Generando diferencias estadísticamente significativas en el índice de frecuencia de accidentes laborales medido en la empresa antes 18,54 y después de su implementación 3,3 . Asumiendo un error $\alpha=0,05$, la significación bilateral es 0,026 misma que es menor a 0,05. El modelo correlacional del modelo de implementación del SG-PRL con las gestiones administrativa, técnica, de talento humano y procesos operativos, a través del análisis de regresión lineal, determino los siguientes resultados:
Se han correlacionado las dimensiones del modelo de Implementación de la gestión de la prevención de riesgos laborales habiéndose obtenido una correlación $R=95,9 \%$. Esto significa que existe una correlación directa y fuerte entre el modelo de gestión de la prevención de riesgos laborales implementado con las gestiones administrativa, técnica, de talento humano y procesos operativos. El coeficiente de determinación de 92,0\%, significa que en un 92 por ciento de las veces, este modelo de gestión de la prevención de riesgos laborales implementado se explica por éstas dimensiones. Con relación al Análisis Beta, se halla que todas las dimensiones son significativas en el sistema de gestión de la seguridad y salud ocupacional del modelo Ecuador. 
Tabla 2. Prueba de los rangos con signo de Wilcoxon

\begin{tabular}{|c|c|c|c|c|c|c|}
\hline \multicolumn{7}{|c|}{ Estadísticos descriptivos } \\
\hline & $\mathrm{N}$ & Media & Desviació & ón típica & Mínimo & Máximo \\
\hline $\begin{array}{l}\text { INDICE FRECUENCIA } \\
\text { ACCIDENTES INICIAL } \\
\text { INDICE FRECUENCIA } \\
\text { ACCIDENTES FINAL }\end{array}$ & 12 & 18,5417 & & 22,33938 & , & 64,10 \\
\hline \multicolumn{7}{|c|}{ Rangos } \\
\hline & & & $\mathrm{N}$ & \multicolumn{2}{|c|}{ Rango promedio } & Suma de rangos \\
\hline $\begin{array}{l}\text { INDICE FRECUENCIA } \\
\text { ACCIDENTES FINAL - INDICE } \\
\text { FRECUENCIA ACCIDENTES } \\
\text { INICIAL }\end{array}$ & $\begin{array}{l}\text { Rangos negati } \\
\text { Rangos positiv } \\
\text { Empates } \\
\text { Total }\end{array}$ & & $\begin{array}{l}6^{9} \\
0^{\circ}\end{array}$ & \multicolumn{2}{|r|}{$\begin{array}{r}3,50 \\
, 00\end{array}$} & $\begin{array}{r}21,00 \\
, 00\end{array}$ \\
\hline \multicolumn{7}{|c|}{$\begin{array}{l}\text { a. INDICE FRECUENCIA ACCIDENTES FINAL < INDICE FRECUENCIA ACCIDENTES INICIAL } \\
\text { b. INDICE FRECUENCIA ACCIDENTES FINAL > INDICE FRECUENCIA ACCIDENTES INICIAL } \\
\text { c. INDICE FRECUENCIA ACCIDENTES FINAL = INDICE FRECUENCIA ACCIDENTES INICIAL } \\
\text { Estadísticos de contraste }{ }^{a}\end{array}$} \\
\hline \multicolumn{7}{|c|}{$\begin{array}{l}\text { INDICE FRECUENCIA ACCIDENTES FINAL - INDICE } \\
\text { FRECUENCIA ACCIDENTES INICIAL }\end{array}$} \\
\hline $\begin{array}{l}\text { Sig. asintót. (bilateral) } \\
\end{array}$ & & & & & & $\begin{array}{r}-2,226^{\circ} \\
, 026\end{array}$ \\
\hline
\end{tabular}

Nota: *Corresponde a los índices de frecuencia anual de accidentes laborales.

Fuente: Elaboración propia.

Tabla 3. Modelo de correlación implementación SG-PRL y dimensiones

\begin{tabular}{|c|c|c|c|c|c|c|c|c|c|c|}
\hline \multicolumn{11}{|c|}{ Coeficientes $^{a}$} \\
\hline \multirow{2}{*}{\multicolumn{3}{|c|}{ Modelo }} & $\begin{array}{l}\text { Coeficientes } \\
\text { estandarizado }\end{array}$ & $\mathrm{nq}$ & $\begin{array}{l}\text { Coeficientes } \\
\text { tipificados }\end{array}$ & \multirow[t]{2}{*}{$t$} & \multirow[t]{2}{*}{ Sig. } & \multicolumn{2}{|c|}{$\begin{array}{l}\text { Intervalo de } \\
95,0 \% \text { para B }\end{array}$} & confianza de \\
\hline & & & $B$ & Error típ. & Beta & & & \multicolumn{2}{|c|}{ Límite inferior } & $\begin{array}{l}\text { Limite } \\
\text { superior }\end{array}$ \\
\hline \multicolumn{3}{|c|}{ (Constante) } & $-11,133$ & 5,367 & & $-2,075$ & ,044 & \multicolumn{2}{|c|}{$-21,949$} &,- 318 \\
\hline \multirow{4}{*}{1} & \multirow{2}{*}{\multicolumn{2}{|c|}{ Gestión Administrativa }} &, 213 &, 100 &, 214 & 2,126 &, 039 & \multicolumn{2}{|c|}{, 011} &, 415 \\
\hline & & & 274 &, 105 &, 272 & 2,617 &, 012 & \multicolumn{2}{|c|}{, 063} &, 485 \\
\hline & \multicolumn{2}{|c|}{$\begin{array}{l}\text { Gestión del Talento } \\
\text { Humano }\end{array}$} &, 216 &, 072 &, 306 & 2,987 &, 005 & \multicolumn{2}{|c|}{, 361} & 070 \\
\hline & \multicolumn{2}{|c|}{ Programas Operativos } &, 521 &, 079 &, 661 & 6,584 &, 000 & \multicolumn{2}{|c|}{, 361} & 680 \\
\hline \multicolumn{11}{|c|}{ a. Variable dependiente: Modelo Implementación Gestión Prevención Riesgos Laborales } \\
\hline \multicolumn{11}{|c|}{ Resumen del modelob $^{b}$} \\
\hline \multirow{2}{*}{$\begin{array}{l}\text { Model } \\
0\end{array}$} & \multirow[t]{2}{*}{$\mathrm{R}$} & \multirow{2}{*}{$\begin{array}{c}\mathrm{R} \\
\text { cuadrado }\end{array}$} & \multirow{2}{*}{$\begin{array}{c}\text { R cuadrado } \\
\text { corregida }\end{array}$} & \multirow{2}{*}{$\begin{array}{l}\text { Error típ. de } \\
\qquad \text { la } \\
\text { estimación }\end{array}$} & \multicolumn{6}{|c|}{ Estadísticos de cambio } \\
\hline & & & & & $\begin{array}{l}\text { Cambio en } \\
\text { R cuadrado }\end{array}$ & $\begin{array}{c}\text { Cambio } \\
\mathrm{F}\end{array}$ & & gl1 & gl2 & $\begin{array}{l}\text { Sig. Cambio } \\
\text { en } \mathrm{F}\end{array}$ \\
\hline 1 &, $959^{\mathrm{a}}$ & ,920 & ,917 & 0,251 & ,290,726 & & 4 & 4 & 44 &, 000 \\
\hline
\end{tabular}

Nota: *Corresponde al modelo de regresión lineal resultante.

Fuente: Elaboración propia. 


\section{CONCLUSIONES}

1. La integración del sistema de gestión de la prevención de riesgos laborales, permite que todos los estamentos de la empresa analicen y entiendan el principio de funcionamiento de todos los componentes que intervienen en la seguridad y ambientes de trabajo.

2. El modelo de implementación del sistema de gestión de la prevención de riesgos laborales es el proceso mediante el cual una organización pone en funcionamiento los procedimientos del sistema de gestión de seguridad y salud laboral con eficacia, permitiendo una disminución de la accidentabilidad laboral.

3. El Sistema de Gestión de Prevención de Riesgos laborales implementado, con la metodología propuesta ha mejorado su índice de eficacia. Se ha pasado de un valor inicial de $53,29 \%$ a $93,52 \%$ en el indicador final, lo cual plantea una diferencia y mejora significativa.

4. En la prueba de los rangos con signo de Wilcoxon, asumiendo un error $\alpha=0,05$, la significación bilateral es 0.026 misma que es menor a 0.05 razón por la cual sí hay diferencias significativamente efectivas, ello implica el poder afirmar que la implementación del Sistema de gestión de prevención de riesgos laborales, en la industria láctea analizada disminuye el índice de frecuencia de accidentes laborales (IF), en dónde el IF medido en la empresa antes de la implementación fue de 18,54 y después de su implementación 3,3.

5. El índice de frecuencia de accidentes referencial en industrias de alimentos y bebidas según el Instituto Ecuatoriano de Seguridad Social es de 5, por tanto al tener un IF de 3,3 tenemos un alto nivel de seguridad laboral.

6. El modelo correlacional es muy significativo e incide en un 92 por ciento de las veces. Es decir el modelo de gestión de la prevención de riesgos laborales implementado se explica por las dimensiones administrativa, técnica, de talento humano y de 'procesos operativos.

\section{REFERENCIAS BIBLIOGRÁFICAS}

[1] Azcuénaga L. (2004). Guía para la Implantación de un sistema de Prevención de Riesgos Laborales.Madrid, España: FC Editorial.

[2] Andino, C. (2005). Reglamento del instrumento andino de seguridad y salud en el trabajo. IE Social, Resolución, 957. En F. Carpio (Ed.), Seguro General de Riesgos del Trabajo. (pp. 54-87). Quito, Ecuador: Publicaciones IESS

[3] Díaz, E. (2013). Condiciones de trabajo en la industria de procesamiento lácteo, eslabón principal de la cadena de valor/Regiones de Los Lagos y Los Ríos. Santiago de Chile: Andros Impresores.

[4] Enríquez,A., \& Sánchez, J. (2008). OHSAS 18001: 2007. Interpretación, aplicación y equivalencias legales, Madrid, España: FC Editorial.

[5] IESS, R. C. (2010). 333 Reglamento para el Sistema de Auditoría de Riesgos del Trabajo"SART". En F. Carpio (Ed.), Seguro General de Riesgos del Trabajo. (pp. 2-34). Quito, Ecuador: Publicaciones IESS.

[6] Peula, M. R. (2014). Envasado y acondicionamiento de productos lácteos. INAE0209.Malaga, España: IC Editorial.

[7] Peltier, T. R. (2013). Information security fundamentals. Boca Raton, FL United States: CRC press.

[8] Rivas, G. P. M., Rivas, R. M., \& Giraldo, J. B. (2015). Correlación de la gestión de riesgos profesionales en la productividad de las empresas del sector cerámico del Valle de Aburrá. Ingenierías USBmed, 5(2), 6-11. http:// revistas.usb.edu.co/index.php/ingUSBmed

[9] Vargas, E. (2010). Estudio de Factibilidad para la creación de una Empresa Productora y Comercializadora de leche en el sector de Mindo Provincia de Pichincha. Memoria para optar al título de Ingeniero empresarial, Facultad de Ciencias Administrativas, Escuela Politécnica Nacional, Quito, Ecuador.

[10] Vidal, E. O., Santiago, J. M., López, R. I. M., \& Delgado, J. C. (2015). Las técnicas de prevención de los riesgos laborales. En C. Molina (Ed.), Tratado de prevención de riesgos laborales: teoría y práctica (pp. 985-1214). Madrid, España: Tecnos. 